\title{
Interference effects in phased beam tracing using exact half-space solutions
}

Matthew A. Boucher, Bert Pluymers, and Wim Desmet

Citation: J. Acoust. Soc. Am. 140, 4204 (2016); doi: 10.1121/1.4971283

View online: http://dx.doi.org/10.1121/1.4971283

View Table of Contents: http://asa.scitation.org/toc/jas/140/6

Published by the Acoustical Society of America

\section{Articles you may be interested in}

Connecting the grain-shearing mechanism of wave propagation in marine sediments to fractional order wave equations

J. Acoust. Soc. Am. 140, (2016); 10.1121/1.4971289

Examination of the reflection properties of sloping terminations to organ pipes

J. Acoust. Soc. Am. 140, (2016); 10.1121/1.4969466

Acoustics from a rectangular supersonic nozzle exhausting over a flat surface

J. Acoust. Soc. Am. 140, (2016); 10.1121/1.4967158

Swim track kinematics and calling behavior attributed to Bryde's whales on the Navy's Pacific Missile Range Facility

J. Acoust. Soc. Am. 140, (2016); 10.1121/1.4967754 


\title{
Interference effects in phased beam tracing using exact half-space solutions
}

\author{
Matthew A. Boucher, ${ }^{\text {a),b) }}$ Bert Pluymers, ${ }^{\text {b) }}$ and Wim Desmet ${ }^{\text {b) }}$ \\ Division PMA, Department of Mechanical Engineering, KU Leuven, BE-3001 Heverlee, Belgium
}

(Received 17 June 2016; revised 11 November 2016; accepted 18 November 2016; published online 8 December 2016)

\begin{abstract}
Geometrical acoustics provides a correct solution to the wave equation for rectangular rooms with rigid boundaries and is an accurate approximation at high frequencies with nearly hard walls. When interference effects are important, phased geometrical acoustics is employed in order to account for phase shifts due to propagation and reflection. Error increases, however, with more absorption, complex impedance values, grazing incidence, smaller volumes and lower frequencies. Replacing the plane wave reflection coefficient with a spherical one reduces the error but results in slower convergence. Frequency-dependent stopping criteria are then applied to avoid calculating higher order reflections for frequencies that have already converged. Exact half-space solutions are used to derive two additional spherical wave reflection coefficients: (i) the Sommerfeld integral, consisting of a plane wave decomposition of a point source and (ii) a line of image sources located at complex coordinates. Phased beam tracing using exact half-space solutions agrees well with the finite element method for rectangular rooms with absorbing boundaries, at low frequencies and for rooms with different aspect ratios. Results are accurate even for long source-to-receiver distances. Finally, the crossover frequency between the plane and spherical wave reflection coefficients is discussed.
\end{abstract} (C) 2016 Acoustical Society of America. [http://dx.doi.org/10.1121/1.4971283]

[MV]

Pages: 4204-4212

\section{INTRODUCTION}

Geometrical acoustics (GA) methods are common tools used to predict the sound in un-built environments, modify the sound in already existing rooms and even in perceptual studies and virtual reality. Since the concept of sound waves is replaced by that of sound rays, ${ }^{1}$ wave behavior such as diffraction, scattering and interference pose unique challenges in GA modeling and are, therefore, studied separately. This work studies empty, rectangular rooms with ideally smooth surfaces and uniform boundary conditions. Under these conditions, diffraction and scattering are not present, isolating the interference phenomenon, which can be calculated using phased geometrical acoustics methods. Whereas previous phased GA studies primarily employ the plane wave reflection coefficient (PWRC), this study provides an assessment of using spherical wave reflection coefficients (SWRCs) in phased beam tracing. ${ }^{2,3}$ Besides the known SWRC from the literature, ${ }^{4}$ two additional SWRCs are presented that are derived from exact, half-space solutions, ${ }^{5,6}$ providing more robust calculations of room transfer functions.

The limitations of the PWRC are well-documented. Lam concludes that the plane wave reflection coefficient gives higher errors for higher absorption and for higher order reflections. ${ }^{4}$ Suh and Nelson conclude that the accuracy of the PWRC deteriorates "for grazing incidence sound or surfaces of high absorption coefficient when the source-receiver distance is less than or equal to a few wavelengths." Replacing the PWRC with the SWRC gives results that

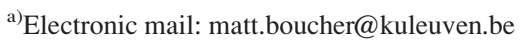

b) Also at Flanders Make, Leuven, Belgium.
}

agree very well with the boundary element method for rectangular rooms, even at low frequencies. ${ }^{4}$ However, Lam reports an oscillation effect at low frequencies or at offresonances in a reverberation room with hard walls. The oscillations are attributed to a $-30 \mathrm{~dB}$ cut-off for image sources that are weak compared to the direct sound and are present for both the PWRC and the SWRC. In this work, no dynamic range is implemented, which removes the oscillations and facilitates error analysis.

Mechel identifies the SWRC used by Lam as the Chien/ Soroka approximation, ${ }^{7}$ which is derived from the Sommerfeld integral, an exact half-space solution for a point source above an infinite plane of arbitrary impedance, originally treated in telecommunications. ${ }^{8}$ In acoustics, the Sommerfeld integral is usually applied in outdoor sound propagation problems where the source and/or receiver are located close to the absorbing ground ${ }^{9}$ or in underwater acoustics, in which the seabed is assumed to have a finite (rather low) impedance. ${ }^{10}$ A separate formulation of the half-space problem is presented by Ochmann, ${ }^{6}$ who uses it to construct a Green's function that takes into account the reflection from an infinite plane of arbitrary impedance, which is then incorporated into the boundary element method. Useful discussions on the different integral representations of the half-space solutions, their development and their approximations, are given by Taraldsen. ${ }^{11,12}$

This work compares four reflection models (two of which are derived from the exact solutions just described) that are incorporated into a phased beam tracing method ${ }^{2,3}$ and discusses several aspects of interference effects in rectangular rooms. The models are tested for various boundary conditions in rooms with different aspect ratios and receivers 
distributed throughout the room, using the finite element method as a reference.

\section{METHOD}

The steady-state sound pressure level (SPL) is calculated in three rectangular rooms using phased geometrical acoustics. Four different reflection coefficients are presented. Finally, the triangular beam tracing method is described, which is used to determine the geometrical quantities needed in the phased GA method.

\section{A. Phased geometrical acoustics}

Phased geometrical acoustics consists of the coherent summation of the direct sound and $N$ image sources, allowing both destructive and constructive interference to occur. ${ }^{13-16}$ It has the form given by

$$
p\left(\mathbf{x}_{r}, \mathbf{x}_{s}\right)=\sum_{n=0}^{N} p_{n}=\sum_{n=0}^{N} A_{n} g_{n}\left(k, r_{n}\right),
$$

where $p$ is the pressure at $\mathbf{x}_{r}$ due a point source located at $\mathbf{x}_{s}$. $g$ is the free-space Green's function where $g(k, r)=e^{-\mathrm{j} k r} /$ $(4 \pi r), k$ is the wavenumber and $\mathrm{j}$ is the imaginary number $\left(\mathrm{j}^{2}=-1\right)$. The direct sound is $p_{0}$ with $A_{0}=1$ and $r_{0}$ $=\left\|\mathbf{x}_{r}-\mathbf{x}_{s}\right\|$. For $n>0, A_{n}$ is the effective reflection coefficient and is, in general, a complex value. It is given by

$$
A_{n}=\prod_{m=1}^{M_{n}} a_{m n}
$$

where $M_{n}$ is the number of reflections experienced by the $n$th image source and $a_{m n}$ is the reflection coefficient at the $m$ th reflection. The propagation distance is $r_{n}=\left\|\mathbf{x}_{r}-\mathbf{x}_{s n}\right\|$, where $\mathbf{x}_{s n}$ is the image source position. Then, $p_{n}$ is the pressure contribution of the $n$th image source.

In order to determine the values of $a_{m n}$, each reflection is interpreted as a half-space problem, in which a point source and receiver are located above an infinite reflecting plane of known impedance, $Z$. The heights of the source and receiver above the reflecting plane are $d_{s}$ and $d_{r}$, respectively. The incident angle relative to the surface normal is $\theta$, and the surfaces of the room are defined by $x=0, y=0$, $z=0, x=L_{x}, y=L_{y}$, and $z=L_{z}$.

For a room with at least one pair of parallel walls, there is an infinite number of valid propagation paths. Therefore, the infinite sum must be truncated. ${ }^{17}$ Instead of fixing a maximum propagation distance or a given reflection order, frequency-dependent stopping criteria are implemented in this work. After all image sources of order $M$ are found, the differences in total sound pressure level, $\triangle$ SPL, and phase of the total pressure, $\Delta \eta$, between $M$ and $M-1$ are evaluated for each frequency. Then, the phased GA model is considered to have converged if both changes are below a threshold for two consecutive reflections. The thresholds are set to $|\Delta \mathrm{SPL}|<0.1 \mathrm{~dB}$ and $|\Delta \eta|<0.01 \mathrm{rad}$. Since these criteria are evaluated for each frequency, adjacent frequencies may require different amounts of reflections to converge, meaning they also have different $N$. This has direct implications for the convergence and computation time, which are discussed in Sec. III C.

\section{B. Reflection models}

This section describes four different reflection models used in Eq. (2). Model 1 uses the plane wave reflection coefficient. Model 2 is the spherical wave reflection coefficient used by Lam. ${ }^{4}$ Models 3 and 4 are SWRCs that are derived from the Sommerfeld and Ochmann exact half-space solutions, respectively. All models assume a locally reacting material that is of infinite extent.

\section{Model 1: Plane wave reflection coefficient}

The plane wave reflection coefficient is often used in phased geometrical acoustics. For example, Suh and Nelson compare a phased image source method using the PWRC with experiments in a rectangular room. ${ }^{5}$ Also, Aretz and Vorländer ${ }^{17}$ compare a similar phased image method with the finite element method in order to assess the effect of various truncation rules and approximate reflection coefficients. Wareing and Hodgson ${ }^{18}$ combine this model with the transfer matrix method to include boundaries described as multilayers of fluid, solid and porous materials. The PWRC is given by

$$
a_{m n} \approx R_{p}\left(\theta_{m n}\right)=\frac{Z_{0} \cos \theta_{m n}-1}{Z_{0} \cos \theta_{m n}+1},
$$

where $Z_{0}=Z / \rho c$ is the normalized surface impedance of the reflecting surface and $\theta_{m n}$ is the incident angle, also equal to the reflected angle. This reflection model is accurate for high frequencies, non-grazing incidence and for low absorbing surfaces. ${ }^{5}$

\section{Model 2: Spherical wave reflection coefficient}

The second reflection coefficient is an approximation to the Sommerfeld solution (see model 3 below), assuming a spherical source impinging upon a planar surface. It is given by

$$
a_{m n} \approx R_{p}+\left(1-R_{p}\right) F\left(w_{m n}\right)
$$

where $F(w)=1+\mathrm{j} w \sqrt{\pi} \mathrm{e}^{-w^{2}} \operatorname{erfc}(-\mathrm{j} w)$ is the boundary loss factor; erfc is the complementary error function. The numerical distance is $w_{m n}=\sqrt{\mathrm{j} k r_{n} / 2}\left(\cos \theta_{m n}+1 / Z_{0}\right)$. This is the Chien/Soroka approximation which is valid for $\left|1 / Z_{0}\right| \ll 1$ and $k r_{n} \gg 1 .^{7}$ It is used to predict the sound attenuation in tunnels ${ }^{19}$ and has been shown to agree well with the boundary element method. ${ }^{4}$ It should be noted that model 2 is formulated for the $\mathrm{e}^{-\mathrm{j} \omega t}$ time convention, so the conjugate impedance should be used.

\section{Model 3: Sommerfeld solution}

The next solution is exact and is given by the so-called Sommerfeld integral, in which a plane wave decomposition of a spherical point source is integrated over all possible 
incident angles. This solution is used to calculate the error for one reflection in a phased GA model. ${ }^{5,17}$ The reflection coefficient using the Sommerfeld integral is given by

$$
a_{m n}=-\frac{\mathrm{j} k}{4 \pi g_{n}} I_{S}\left(k, \phi_{m n}, \psi_{m n}, Z_{0}\right),
$$

where

$$
I_{S}=\int_{\Gamma_{\beta}} J_{0}(k \phi \sin \beta) \mathrm{e}^{-\mathrm{j} k \psi \cos \beta} R_{p}(\beta) \sin \beta \mathrm{d} \beta,
$$

and $J_{0}$ is the Bessel function of order zero and $R_{p}(\beta)$ is the plane wave reflection coefficient with incident angle $\beta$. The reflected pressure is expressed in terms of the sum of the heights of the source and receiver above the reflecting plane, $\psi=d_{s}+d_{r}$, and the in-plane distance from source to receiver, $\phi$. These values are calculated simply as $\psi_{m n}$ $=r_{n} \cos \theta_{m n}$ and $\phi_{m n}=r_{n} \sin \theta_{m n}$. The domain of integration, $\Gamma_{\beta}$, includes all angles on the interval $(0, \pi / 2)$, including complex values. In practice, the domain is split into two integrals, where the integration path follows $0 \rightarrow \pi / 2 \rightarrow \pi / 2$ $+\mathrm{j} \infty$. The reader is referred to the Appendix in Suh and Nelson $^{5}$ for more details. The second integral is tested for convergence by increasing its truncation limit and comparing successive evaluations.

\section{Model 4: Ochmann solution}

The other exact solution used in the present work, equivalent to model 3 , is given by Ochmann, ${ }^{6}$ which he uses to construct a half-space Green's function that is then used in the boundary element method. The exact reflection coefficient may be defined as

$$
a_{m n}=1-\frac{k}{2 \pi Z_{0} g_{n}} I_{O}\left(k, \phi_{m n}, \psi_{m n}, Z_{0}\right),
$$

where

$$
I_{O}=\int_{-\infty}^{0} \frac{\mathrm{e}^{-\mathrm{j} k \sqrt{\phi^{2}+(\psi+\mathrm{j} \zeta)^{2}}}}{\sqrt{\phi^{2}+(\psi+\mathrm{j} \zeta)^{2}}} \mathrm{e}^{\mathrm{k} \zeta / \mathrm{Z}_{0}} \mathrm{~d} \zeta .
$$

The first term in Eq. (7) is the contribution from an image source assuming a perfectly rigid reflecting surface. The second term is a line of image sources located at complex coordinates. Since $\psi$ is in the direction of the surface normal, $\mathrm{j} \zeta$ has the effect of adding an imaginary part to $d_{s}+d_{r}$. The factor $\mathrm{e}^{k \zeta / Z_{0}}$ is a decaying amplitude term since $\zeta \leq 0$. Ochmann suggests Gauss-Laguerre quadrature to evaluate $I_{O}$ when $\Im\left\{\mathrm{j} k / Z_{0}\right\}>1$. However, this is a high-frequency condition where model 1 , in many cases, already performs well. Di and Gilbert ${ }^{20}$ suggest truncation of the integral at a value of $\zeta_{D G}=-\lambda\left[\left(\Re\left[Z_{0}\right]^{2}+\Im\left[Z_{0}\right]^{2}\right) /\left(\Re\left[Z_{0}\right]\right)\right]$, where $\lambda$ is the wavelength. $\zeta_{D G}$ is independent of the in-plane distance $\phi$, but Ochmann clearly shows that the integrand exhibits a sharp peak at $\zeta=-\phi$ when the source and receiver are located close to the reflecting surface. Therefore, in this work, a more conservative lower limit is used, given by $\zeta_{\text {lower }}$ $=-\phi+\zeta_{D G}$, and Gaussian quadrature is used.

\section{Triangular beam tracing}

The geometrical quantities needed in Eq. (1), such as $r_{n}$, $\theta_{m n}, \phi_{m n}$, and $\psi_{m n}$, are found using triangular beam tracing, ${ }^{2,3}$ implemented by the authors in the MATLAB ${ }^{21}$ environment. An omnidirectional source is modeled through geodesic subdivision of an icosahedron. Each edge is divided into $u$ segments, so that it yields $u^{2}$ triangular beams. Since an icosahedron has 20 faces, this leads to a total of $20 u^{2}$ beams that cover the entire solid angle of $4 \pi$. Since there are no gaps between the beams, the direct sound is always registered.

The method is called approximate beam tracing, because the center ray of each beam is traced throughout the room, and the beam is not split if it intersects more than one surface. At each reflection, the apex of the image beam corresponds to the location of an image source, which is considered valid if the receiver lies inside the viewing angle of the image beam. More details on approximate triangular beam tracing can be found in the works of Lewers, ${ }^{2}$ Wareing and Hodgson, ${ }^{18}$ and Jeong. ${ }^{3}$

The lack of beam splitting can result in the scanning error, in which a valid propagation path can be missed or an invalid one can be counted. ${ }^{2}$ A practical way of limiting this effect is by ensuring that the edge length of the base of each beam is less than the minimum dimension of the room, $L_{\text {min }}$. At a propagation distance of $c T_{60}$, where $T_{60}$ is the reverberation time, the edge length of an icosahedron is approximately $1.05 c T_{60} \approx c T_{60}$. Enforcing the edge length of each beam to be around $L_{\mathrm{min}} / 2$ leads to

$$
u=\left\lceil\frac{2 c T_{60}}{L_{\min }}\right\rceil
$$

where $L_{\min }$ is the minimum of $L_{x}, L_{y}$, and $L_{z}$ and where $\lceil\cdot\rceil$ is the ceiling function. This criterion does not guarantee convergence. However, it is a conservative value (resulting in a large amount of beams) that effectively limits the scanning error, facilitating the evaluation of the different reflection models.

\section{RESULTS}

The sound pressure level calculated with four reflection models using Eq. (1) is compared with the finite element method (FEM) calculated with the LMS Virtual.Lab R12 software. The FEM is chosen as a reference solution, because it is a standard numerical tool for low frequency acoustics problems. Also, since it is based on a discretization of the weak formulation of the Helmholtz equation, ${ }^{22}$ all wave effects, including interference, are included correctly. The FEM Adaptive Order algorithm is applied and allows accurate and fast calculations. ${ }^{23}$

The geometry of the rooms studied is summarized in Table I. A proportionate room $\left(7 \times 5 \times 3 \mathrm{~m}^{3}\right)$ is taken from the work of Aretz. ${ }^{17}$ Rooms with larger aspect ratios such as the long $\left(10 \times 3 \times 3 \mathrm{~m}^{3}\right)$ and flat $\left(10 \times 10 \times 3 \mathrm{~m}^{3}\right)$ rooms 
TABLE I. Configurations of different rooms studied.

\begin{tabular}{lccc}
\hline \hline Room & Size $\left(\mathrm{m}^{3}\right)$ & $\mathrm{x}_{s}(\mathrm{~m})$ & $\mathrm{x}_{r}(\mathrm{~m})$ \\
\hline 1 & $7 \times 5 \times 3$ & $(5,3.5,1.6)$ & $(2.5,2,1.2)$ \\
2 & $10 \times 3 \times 3$ & $(0.5,1.5,2)$ & $(9.5,1.5,1.8)$ \\
3 & $10 \times 10 \times 3$ & $(0.5,5,1.5)$ & $(9.5,5,1.8)$ \\
\hline
\end{tabular}

used by Wareing are also tested. ${ }^{18}$ These rooms are chosen, because due to their small volume relative to auditoriums and concert halls, interference effects are expected to play a large role. The source and receiver locations for the three rooms are shown in Table I. Three sets of impedance boundary conditions are applied to each room, corresponding to varying amounts of absorption. For each level of absorption, impedance values with phase angles $\mu$ of $0^{\circ}$ and $\pm 30^{\circ}$ are applied. The values are summarized in Table II.

Some important parameters of the GA calculations are shown in Table III. The reverberation time is calculated using the Eyring equation. The Schroeder frequency is given by $f_{S} \approx 2000 \sqrt{T_{60} / V}$, where $V$ is the total volume of the room. The random incidence absorption coefficient for each set of boundary conditions $\mathrm{A} / \mathrm{B} \pm, \mathrm{C} / \mathrm{D} \pm$, and $\mathrm{E} / \mathrm{F} \pm$ are roughly the same. This means that boundary conditions within each set have almost identical values for $T_{60}$ and $f_{S}$. The number of beams is given by $N_{b}=20 u^{2}$ where $u$ is determined by Eq. (9).

At every reflection, model 4 is used to evaluate $\triangle \mathrm{SPL}$ and $\Delta \eta$, because in general, spherical wave reflection coefficients converge slower than the PWRC. ${ }^{4}$ Then, after a certain number of reflections $M_{\max }$, all frequencies satisfy the convergence criteria specified in Sec. II A, which ends the simulation. Lower absorption cases require higher number of reflections before reaching the steady state, because less energy is dissipated at each reflection. Taking room 3 as an example, boundary condition B results in $M_{\max }=14\left(N_{\max }\right.$ $=3,914)$ while boundary condition $\mathrm{F}$ results in $M_{\max }=62$ $\left(N_{\max }=303,534\right)$.

The error used in this work is the absolute error of the SPL of the GA model relative to the FEM solution, averaged over a certain frequency range. It is given by

$$
\epsilon=\frac{1}{f_{u b}-f_{l b}+1} \sum_{f}\left|\operatorname{SPL}_{\mathrm{GA}}(f)-\operatorname{SPL}_{\mathrm{FEM}}(f)\right|,
$$

where $f_{l b}$ and $f_{u b}$ are the lower and upper bounds of the frequency range, respectively. The frequencies are calculated at integer values within the interval, including the bounds. This

TABLE II. Different boundary conditions tested.

\begin{tabular}{lccc}
\hline \hline & $Z_{0}$ & $\mu\left(^{\circ}\right)$ & $\alpha$ \\
\hline $\mathrm{A}$ & 5.3 & 0 & 0.70 \\
$\mathrm{~B} \pm$ & $3.77 \pm \mathrm{j} 2.17$ & \pm 30 & 0.70 \\
$\mathrm{C}$ & 9.6 & 0 & 0.50 \\
$\mathrm{D} \pm$ & $7.08 \pm \mathrm{j} 4.09$ & \pm 30 & 0.50 \\
$\mathrm{E}$ & 19.8 & 0 & 0.30 \\
$\mathrm{~F} \pm$ & $14.69 \pm \mathrm{j} 8.48$ & \pm 30 & 0.30 \\
\hline \hline
\end{tabular}

TABLE III. Room parameters.

\begin{tabular}{|c|c|c|c|c|c|c|c|c|c|}
\hline \multirow[b]{2}{*}{$\alpha$} & \multicolumn{3}{|c|}{ Room 1} & \multicolumn{3}{|c|}{ Room 2} & \multicolumn{3}{|c|}{ Room 3} \\
\hline & $\begin{array}{r}T_{60} \\
(\mathrm{~s})\end{array}$ & $\begin{array}{c}f_{S} \\
(\mathrm{~Hz})\end{array}$ & $N_{b}$ & $\begin{array}{r}T_{60} \\
\text { (s) }\end{array}$ & $\begin{array}{c}f_{S} \\
(\mathrm{~Hz})\end{array}$ & $N_{b}$ & $\begin{array}{c}T_{60} \\
(\mathrm{~s})\end{array}$ & $\begin{array}{c}f_{S} \\
(\mathrm{~Hz})\end{array}$ & $N_{b}$ \\
\hline 0.7 & 0.10 & 61 & 10,580 & 0.09 & 62 & 8,000 & 0.13 & 41 & 16,820 \\
\hline 0.5 & 0.17 & 81 & 32,000 & 0.15 & 82 & 24,500 & 0.22 & 54 & 50,000 \\
\hline 0.3 & 0.33 & 113 & 118,580 & 0.29 & 114 & 92,480 & 0.42 & 75 & 188,180 \\
\hline
\end{tabular}

error over the range of $20 \mathrm{~Hz}$ to $\left\lceil 3 f_{S}\right\rceil$ is notated by $\epsilon_{1}$, and $\epsilon_{2}$ is the error over $\frac{1}{3}$-octave bands. A special case of Eq. (10) is the absolute error evaluated at one frequency, $\epsilon_{0}$.

In this section, the main results are presented. First, the average absolute error of the SPL from $20 \mathrm{~Hz}$ to $3 f_{S}$ are shown. Then, the SPL is given for two cases, accompanied by either the absolute error averaged over $\frac{1}{3}$-octave bands or at $1 \mathrm{~Hz}$ intervals. The models are compared for different source/receiver combinations in Sec. III B. In Sec. III C, convergence and computation times are discussed. The main reason for the improvements when using exact half-space solutions is described in Sec. III D. Finally, the crossover frequency between plane and spherical wave reflection coefficients is presented in Sec. III E.

\section{A. SPL and accuracy}

The accuracy of the phased GA models for room 1 with nine different boundary conditions is summarized in Fig. 1, showing the values for $\epsilon_{1}$. As expected, the error for model 1 is greater for higher absorption and complex impedance values. Models 3 and 4 reduce the error of model 1 by an average of $1.0 \mathrm{~dB}$. Model 2 agrees well with models 3 and 4 , with noticeable differences mostly for $\alpha=0.7(\mathrm{~A} / \mathrm{B} \pm)$.

Rooms 2 and 3 give similar results, which are summarized in Table IV. The values shown are the averages of $\epsilon_{1}$ over the nine boundary conditions. Model 1 , in general, shows $1-2 \mathrm{~dB}$ error with respect to the FEM, with increasing errors for the long and flat rooms. Models 2, 3, and 4 usually show improvements over model 1 of $1 \mathrm{~dB}$ or more.

The best performance of model 1 in this study is for room $1 \mathrm{E}$ (room 1 with boundary condition $\mathrm{E}$ ), which represents the highest real impedance studied in the room with the lowest aspect ratio. The frequency response of models 1,2 ,

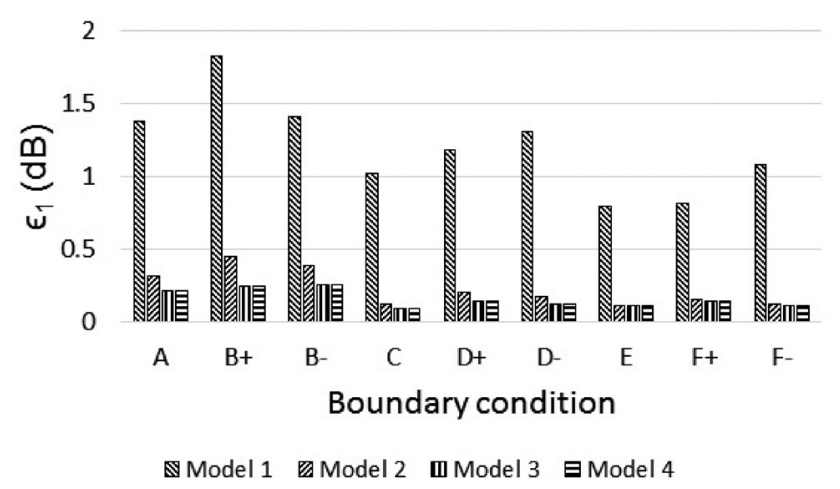

FIG. 1. Absolute error of SPL (ref. FEM) averaged from $20 \mathrm{~Hz}$ to $3 f_{S}$ in room 1. 
TABLE IV. $\epsilon_{1}(\mathrm{~dB})$ averaged over nine boundary conditions.

\begin{tabular}{lccc}
\hline \hline Model & Room 1 & Room 2 & Room 3 \\
\hline 1 & 1.2 & 1.6 & 1.9 \\
2 & 0.2 & 0.3 & 0.3 \\
$3 / 4$ & 0.2 & 0.2 & 0.3 \\
\hline \hline
\end{tabular}

and 4 are compared to FEM in Fig. 2(a)-model 3 is excluded, because it is coincident with model 4 . The absolute error in SPL averaged over $\frac{1}{3}$-octave bands is shown in Fig. 2(b). All phased GA models are, in general, quite accurate, indicated by $\epsilon_{1}$ values of $0.8 \mathrm{~dB}$ for model 1 and $0.1 \mathrm{~dB}$ for models 2 through 4 . However, model 1 can exhibit large deviations in accuracy with a maximum absolute error of $\epsilon_{0}=11.4 \mathrm{~dB}$ at $f=90 \mathrm{~Hz}$. Remarkably, the absolute error for model 2 is only $1.1 \mathrm{~dB}$ at this frequency; models 3 and 4 are better still $\left(\epsilon_{0}=0.7 \mathrm{~dB}\right)$. Model 1 clearly shows higher error for lower $\frac{1}{3}$-octave bands, a trend not present for models $2-4$ where $\epsilon_{2} \leq 0.2 \mathrm{~dB}$ for all $\frac{1}{3}$-octave bands. This can be considered excellent agreement between the reference solution and models 2-4. Even in this case where model 1 performs quite well, models 2 through 4 reduce $\epsilon_{1}$ by $0.7 \mathrm{~dB}$.

A unique aspect of phased geometrical acoustics is that low SPL is correlated with higher error. ${ }^{4}$ From a phased GA
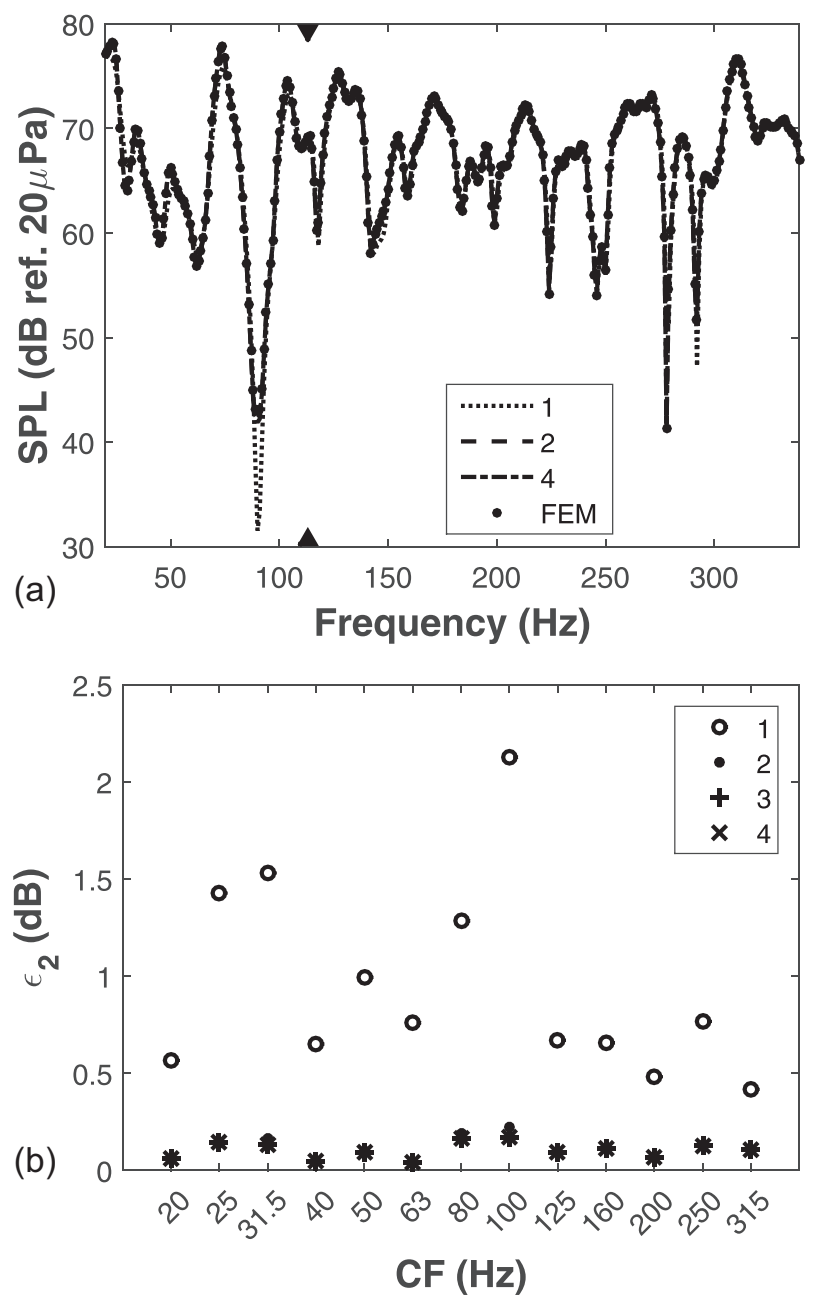

FIG. 2. For room 1E: (a) Sound pressure level and (b) Absolute error of SPL (ref. FEM) averaged over $\frac{1}{3}$-octave bands. $\boldsymbol{\Delta}$ and $\boldsymbol{\nabla}$ indicate $f_{S}=113 \mathrm{~Hz}$. perspective, this is when the most destructive interference occurs among the image sources. This is clearly shown for the case of room 2C. The SPL is shown in Fig. 3(a), while the absolute error in SPL is shown in the upper part of Fig. 3(b). The three frequencies with very low SPL, occurring at 116, 178, and $219 \mathrm{~Hz}$, correspond to peaks in error. Although the effect is still present using model 4, the errors are greatly reduced; likewise for models 2 and 3 .

Compared to $178 \mathrm{~Hz}$, the absolute error for model 1 is actually higher at $179 \mathrm{~Hz}\left(\epsilon_{0}=9.8 \mathrm{~dB}\right)$. This represents a slight shift of the relative minimum of the SPL using model 1. The maximum error of models 2-4 occurs at the relative minimum of the FEM solution, indicating the shift is not present when using an SWRC. This shift is also observed at 197 and $237 \mathrm{~Hz}$ for room $2 \mathrm{C}$ and 238, 291, and $329 \mathrm{~Hz}$ for room 2E. However, since this shift is not observed at all relative minima, it is attributed to the cumulative error of model 1 and not to any physical phenomenon.

Although it is reported that the error at off-resonances may not be too important in practice, ${ }^{4}$ the error at offresonances can have a large effect on the band-averaged values, even above the Schroeder frequency. Continuing with the room $2 \mathrm{C}$ example, the error of model 1 at $116 \mathrm{~Hz}$ results in $\epsilon_{2}=1.8 \mathrm{~dB}$ for the $125 \mathrm{~Hz} \frac{1}{3}$-octave band, while the
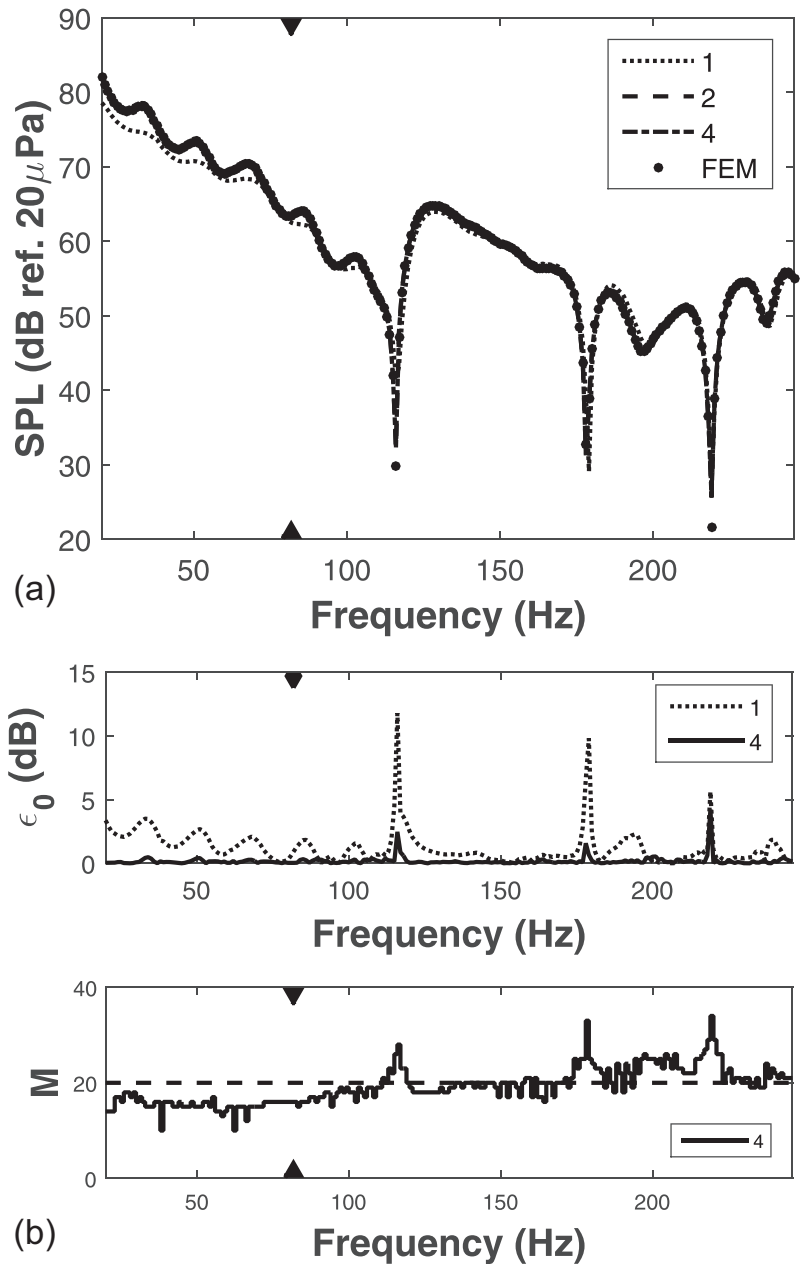

FIG. 3. For room 2C: (a) Sound pressure level. (b) Absolute error of SPL (ref. FEM) and number of reflections $M$ (horizontal line is $\bar{M}=20$ ). $\boldsymbol{\Delta}$ and $\boldsymbol{\nabla}$ indicate $f_{S}=82 \mathrm{~Hz}$. 
two off-resonances occurring in the $200 \mathrm{~Hz} \frac{1}{3}$-octave band result in $\epsilon_{2}=1.2 \mathrm{~dB}$. On the other hand, if the results are energy-averaged into $\frac{1}{3}$-octave bands first and then the absolute difference between model 1 and FEM is taken, the differences are reduced to 0.9 and $0.6 \mathrm{~dB}$, respectively. In either case, these differences may be audible to some listeners, since the just-noticeable-difference for broadband signals is $0.5-1 \mathrm{~dB} .^{24,25}$

It is interesting to note that the correlation between error and SPL is present at other frequencies as well, particularly at $33,51,68,86$, and $103 \mathrm{~Hz}$ for model 1 . The higher error is less pronounced for model $4\left(\epsilon_{0}<0.5 \mathrm{~dB}\right.$ from 20 to $100 \mathrm{~Hz}$ ). Finally, more reflections are required to accurately predict destructive interference, which is shown in Fig. 3(b).

\section{B. Accuracy versus source/receiver position}

To test the spatial dependence of the error, a grid of receivers is analyzed in rooms 1 and 2 for boundary condition $\mathrm{D}-$. The receivers are located at $0.5<x<6.5 \mathrm{~m}$ and $0.5<y<4.5 \mathrm{~m}$ at a height of $z=1.2 \mathrm{~m}$ in room 1 and at $0.5<x<9.5 \mathrm{~m}$ and $0.5<y<2.5 \mathrm{~m}$ at a height of $z=1.8 \mathrm{~m}$ in room 2. The spatial steps are $\Delta x=\Delta y=0.5 \mathrm{~m}$, yielding 117 and 95 receivers for rooms 1 and 2, respectively. The source positions are the same as before. Model 3 is excluded from the analysis due to the large computation time.

The error in SPL for model 1 in room 1, averaged over all receivers and frequencies is $1.2 \mathrm{~dB}$. This error is reduced by around $1 \mathrm{~dB}$ if models 2 or 4 are used, resulting in errors of 0.2 and $0.1 \mathrm{~dB}$, respectively. An even larger reduction in error is found for room 2 with errors of $1.5 \mathrm{~dB}$ for model 1 and $0.3 \mathrm{~dB}$ for models 2 and 4 .

An interesting approach to analyze the error as a function of space is presented by Jeong ${ }^{26}$ who plots the error versus the source-to-receiver distance $r_{0}$. The motivation for this is to determine how the error changes based on different direct-to-reverberant (D-R) energy ratios. This knowledge can be advantageous when optimizing loudspeaker locations in mixing rooms or seating arrangements in auditoriums. The direct sound is known exactly by the Green's function $g_{0}$. Therefore, all the error is concentrated in the reflected sound $\sum_{n=1}^{N} p_{n}$. As $r_{0}$ increases, the direct sound energy decreases, the reflected sound plays a larger role, and the error in the reflections contributes more.

This effect is shown in Fig. 4 for room 1. Discrete values represent the error for one source/receiver combination, while the lines give the best fit to the scattered data. The error grows for models 1,2 , and 4 for larger $r_{0}$, just as shown by Jeong. What is interesting here is that for models 2 and 4, not only is the error reduced but so are the slopes of the linear fits $(0.17,0.021$, and 0.021 for models 1,2 , and 4 , respectively). This means that for large $r_{0}$, models 2 and 4 are less susceptible to increased errors due to source/receiver locations.

To explain this, consider the receivers that are furthest away from the source. These receivers are on the boundary of the grid, so they are close to a reflecting wall (small $\psi$ ) and have large in-plane distances (large $\phi$ ), relative to the other receivers. Since these two conditions are both taken

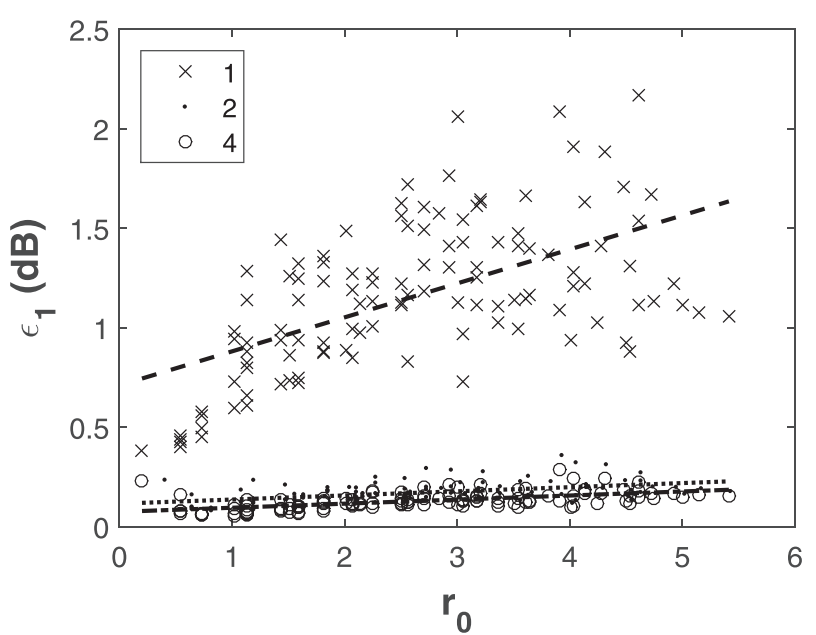

FIG. 4. Absolute error of SPL (ref. FEM) averaged from $20 \mathrm{~Hz}$ to $3 f_{S}$ versus source-to-receiver distance in room 1D-. Dash, dot and dash-dot lines are best fits for particular source-receiver combinations for models 1, 2, and 4, respectively.

into account by model 2 and 4, the dependence of the error on $r_{0}$ is greatly reduced, leading to smaller slopes. The effect is also found for the grid of receivers in room 2, where the slopes of the best fits are $0.15,0.035$, and 0.038 for models 1,2 , and 4 , respectively.

\section{Convergence}

It is reported in the literature that spherical wave reflection coefficients are more susceptible to convergence problems ${ }^{4}$ and that convergence is slower at off-resonances. ${ }^{26}$ This poses a problem when using models 2,3 , or 4 , which is that they can in general require more reflections and, thus, longer computation times, not to mention the additional time to calculate the infinite series in model 2 or to perform the numerical integration of models 3 and 4 . These issues can be alleviated by the frequency-dependent convergence criteria given in Sec. II A. After each reflection, converged frequencies are left out of the calculation procedure, eliminating unnecessary calculations.

Frequency-independent stopping criteria, such as a maximum reflection order or propagation distance, can lead to frequency-dependent error. As shown in Fig. 3(b), the number of reflections needed to converge are shown for room $2 \mathrm{C}$ along with a horizontal line at $\bar{M}=20 . \bar{M}$ represents the reflection order for which the average propagation distance is $c T_{60}$, assuming $\bar{M} \bar{l}=c T_{60}$, where $\bar{l}=4 \mathrm{~V} / \mathrm{S}$ is the mean free path length between diffuse reflections; ${ }^{1} S$ is the total surface area of the room boundaries. For this case, implementing such a cut-off at $\bar{M}$ would not change the results at low frequencies, because they have already converged. Instead, a systematic error would occur at frequencies where more destructive interference occurs, which are largely focused in the $200 \mathrm{~Hz} \frac{1}{3}$-octave band.

Some general properties of the frequency-dependent stopping criteria are shown in Fig. 5 for the case of room $3 \mathrm{~F}$. The time shown is that which is required at each reflection. The number of image sources grows for higher $M$, which means that the computation time curves grow exponentially, 


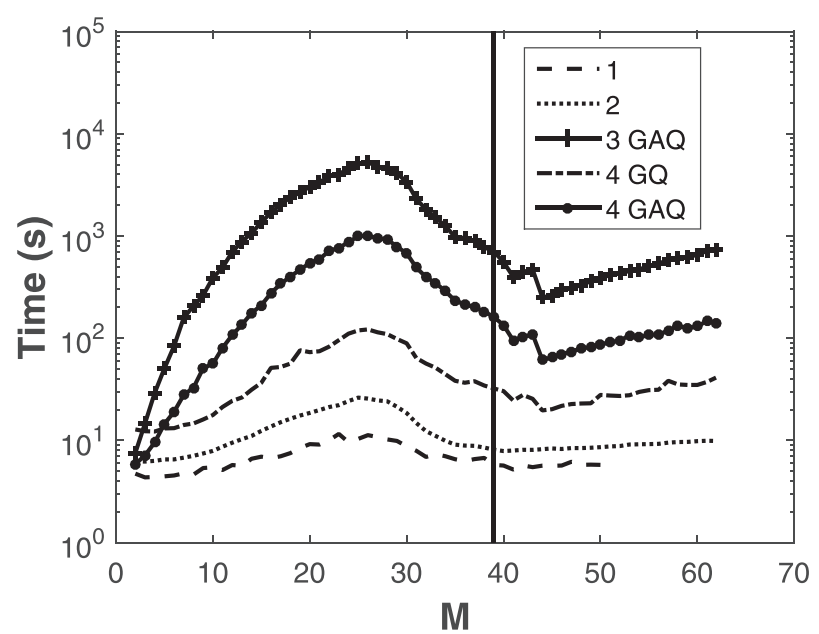

FIG. 5. Computation time per reflection for room $3 \mathrm{~F}(\bar{M}=39)$.

since no frequencies have yet converged. After enough frequencies have converged, the computation time starts to decrease. The increase for models 2,3 , and 4 after $M=45$ is due to the slow convergence of a few remaining frequencies. The most important point is that use of frequency-dependent stopping criteria ensures the convergence of all frequencies but avoids exponential growth of the computation time curve for all $M$.

Also shown in Fig. 5 is the large differences in computation time among the models. Of course, models 2, 3, and 4 take longer than model 1 . For model 2, it is due to the infinite series of the complementary error function. For models 3 and 4 , it is due to the numerical integration at each reflection. Models 3 and 4 using global adaptive quadrature (GAQ) take the most time and are not recommended. However, model 4 lends itself quite nicely to standard Gaussian quadrature (GQ), resulting in about an order of magnitude increase in computation time relative to model 1 . The computation time of model 2 falls in between that of model 1 and model 4 (GQ). All results in this work for model 4 use Gaussian quadrature with 20 integration points per wavelength on $(0,-\phi)$ and six integration points per wavelength on $\left(-\phi, \zeta_{\text {lower }}\right)$. These parameters give virtually identical results as model 3 using global adaptive quadrature.

\section{Magnitude and phase shift upon reflection}

It is clear from the previous results that exact half-space solutions perform much better than the plane wave reflection coefficient. This is because they form a true half-space Green's function, $G=g_{0}+A_{1} g_{1}$, where $A_{1}$ is determined by Eqs. (5) or (7), for a point source above a reflecting plane of arbitrary impedance. Therefore, $G$ satisfies both the Helmholtz equation with a point source excitation as well as the impedance condition on the reflecting surface, i.e., $G=(\mathrm{j} Z / \rho \omega)(\partial G / \partial n)$, where $\partial / \partial n$ is the derivative relative to the surface normal. The Sommerfeld solution satisfies this by summing the contributions of plane wave reflections that include all incident angles (including complex ones). The Ochmann solution satisfies this through a summation of image sources located at complex coordinates.
When these half-space Green's functions are formulated into reflection coefficients, the reflection coefficient can be interpreted as the exact strength of the image source. In this way, each successive reflection becomes a new half-space problem with known strength of a point source above the next reflecting surface. The plane wave solution only satisfies the above conditions for a perfectly rigid $(Z=\infty)$ or soft $(Z=0)$ boundary; in all other cases, model 1 yields a finite error at every reflection.

It is errors in both magnitude and phase that lead to the propagation error of model 1. To illustrate this, Fig. 6 shows the differences between the four reflection models for the reflection with the $z=0$ surface in room $1(\psi=2.8 \mathrm{~m}$ and $\phi \approx 2.9 \mathrm{~m}$ ) with boundary condition A (purely real impedance with $\alpha=0.7$ ). For $\mu=0$, model 1 predicts no phase shift, but this is not correct; the other models do give a phase shift. This results in an error of the reflected SPL of $\geq 0.1 \mathrm{~dB}$ for $f \leq 249 \mathrm{~Hz}$. Lower absorption gives better results, lowering this frequency to 181 and $116 \mathrm{~Hz}$ for boundary conditions $\mathrm{C}$ and $\mathrm{E}$, respectively. When the direct sound is added to the reflected sound as in Eq. (1), the error is reduced at some frequencies and elevated at others due to interference. This results in errors as high as $0.1 \mathrm{~dB}$ up to 534,456 , and $213 \mathrm{~Hz}$ for boundary conditions A, C, and E, respectively. Model 2 predicts the magnitude and phase shift much closer to the exact half-space solutions. The error in the reflected pressure is $\geq 0.1 \mathrm{~dB}$ for $f \leq 60 \mathrm{~Hz}$ and $f \leq 28 \mathrm{~Hz}$ for boundary conditions $\mathrm{A}$ and $\mathrm{C}$. For $\mathrm{E}$, the error is $<0.1 \mathrm{~dB}$ for the entire audible frequency range. When the direct sound is also considered, the error in SPL using model 2 is less than $0.1 \mathrm{~dB}$ for all audible frequencies for boundary conditions A, $\mathrm{C}$, and $\mathrm{E}$.

This is only the error for one reflection, which means error propagates at each additional reflection and for longer propagation distances. ${ }^{4}$ Unfortunately, since the error is correlated between reflections through Eq. (2), statistical error propagation techniques that are used for energetic quantities cannot be applied. ${ }^{27}$
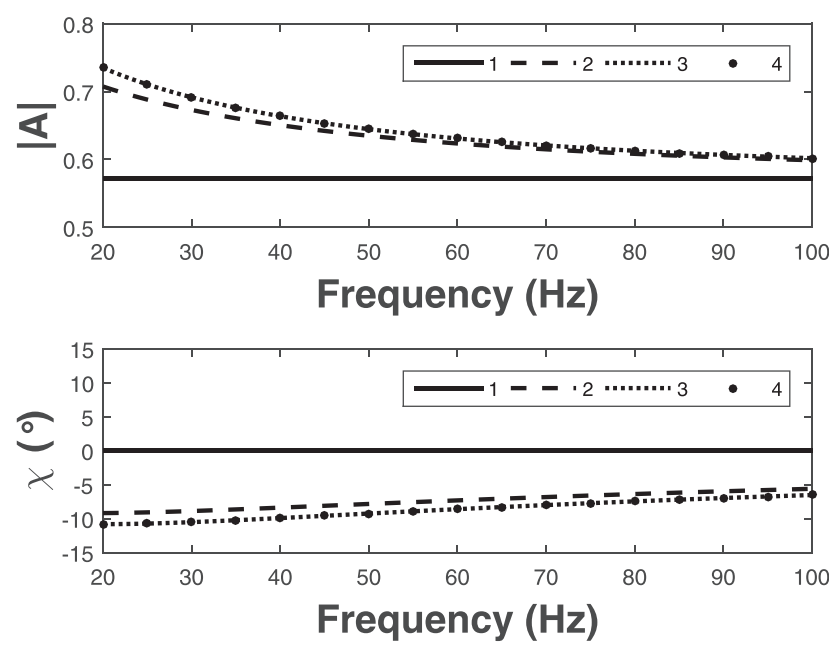

FIG. 6. Magnitude and phase of the reflection coefficient for boundary condition A using four reflection models $(\psi=2.8 \mathrm{~m}$ and $\phi \approx 2.9 \mathrm{~m})$. 


\section{E. Crossover frequency}

In addition to the previous comparisons, a more general question related to geometrical acoustics is that of the crossover frequency. The high frequency region in a room is sometimes assumed to be above the Schroeder frequency. However, as stated by Vorländer, this is not a robust limit between low frequency wave models and high frequency geometrical models. ${ }^{27}$ Aretz suggests $2 f_{S}$ as an acceptable limit. ${ }^{17}$

While the crossover frequency between geometrical and full wave models may be the eventual goal, a practical way to get to this goal may be to identify the crossover frequency for different wave phenomena. Related to the present work, a crossover frequency for interference effects can be defined, above which model 1 differs from models 2 or 4 by less than $1 \mathrm{~dB}$, averaged over a $\frac{1}{3}$-octave band. Because of the computational demand of the FEM, model 4 now becomes the reference, so that $\epsilon=\left[1 /\left(f_{u b}-f_{l b}+1\right)\right] \sum_{f}\left|\operatorname{SPL}_{1}(f)-\mathrm{SPL}_{4}(f)\right|$, where $\mathrm{SPL}_{1}$ is the SPL using model 1 (results are quite similar if model 2 is used as the reference).

Figure 7 shows this error for the room configurations given in Table I with boundary condition $\mathrm{F}+$ from $20 \mathrm{~Hz}$ to $6 f_{S}$. From the above definition, the crossover frequency for room 1 is around the Schroeder frequency. However, for the disproportionate room 2 , the error fluctuates around $1 \mathrm{~dB}$ until around $5 f_{S}$, suggesting a much higher crossover frequency. In room 3 , the error is still around $1 \mathrm{~dB}$ even at $6 f_{S}$. The crossover frequency due to interference effects is, of course, strongly dependent upon the room shape and the boundary condition. The error of model 1 also shows a strong dependence on the source-to-receiver distance, as described in Sec. III B. It seems a difficult task to determine a crossover frequency that takes all of this into account. Finally, should a value other than $1 \mathrm{~dB}$ be chosen, the crossover frequency will change based on the desired accuracy, which may be based on the particular application.

\section{DISCUSSION}

Although diffraction effects are absent in rectangular rooms with uniform boundary conditions and interference

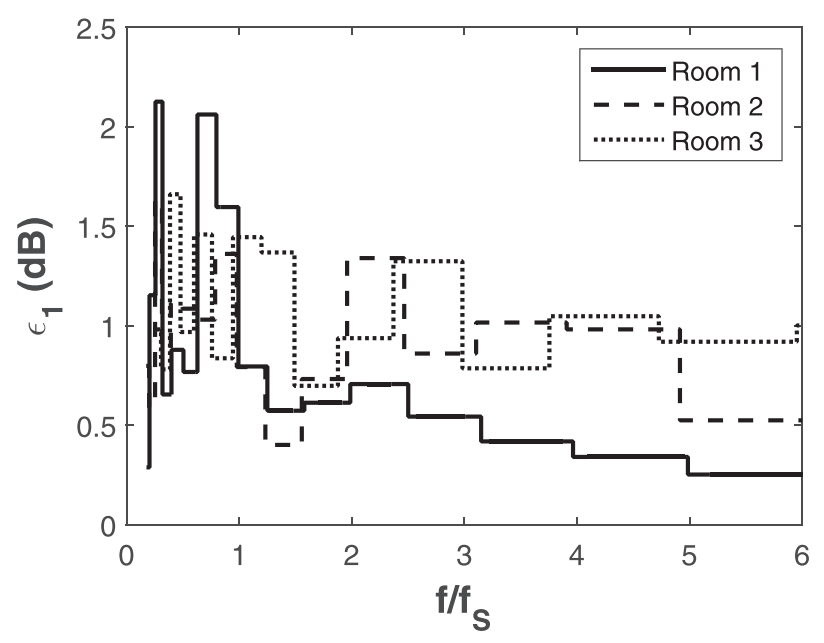

FIG. 7. Absolute error in SPL of model 1 (ref. model 4), averaged over $\frac{1}{3}$ octave bands for boundary condition $\mathrm{F}+$. The frequency axis is normalized by the Schroeder frequency. effects are predicted as accurately as possible, some error still remains, particularly below the Schroeder frequency, for complex impedance values and at off-resonances. The error due to a finite dynamic range $e^{4}$ or a maximum propagation distance $^{17}$ is not applicable due to the convergence criteria defined in Sec. II A. For off-resonances, some of the remaining error may be more noticeable when the level of the simulation error is comparable to the predicted SPL. ${ }^{26}$ The scanning error may also be a source of the remaining error; however, the large amount of beams used in this work makes that unlikely. A more likely possibility is that the finite dimensions of the reflecting surfaces play a role, especially when a source or receiver is located close to an edge. One way to cope with this is to assume that the radiation impedance of the finite surface is equal to the average value of the baffled surface and the infinite surface. ${ }^{28}$ Although this improves the results at low frequencies, some errors are introduced for higher frequencies.

When the reflecting surfaces in a room are assumed infinite, model 2 gives similar improvement as models 3 and 4 but with less computational effort. However, model 2 is still an approximation, meaning that models 3 and 4 are more robust against errors for small $\psi$ and large $\phi$ and $|\mu|$. Therefore, an exact half-space solution can be used for a difficult source/receiver combination or higher absorbing boundaries with large impedance phase angles. In general, model 1 should only be used when the errors are deemed to be within acceptable limits or when computational effort is the main concern, as is the case for real-time applications. For off-line calculations, the steady-state SPL using model 1 should, in most cases, be checked against an SWRC to evaluate its validity.

The cases analyzed in this work include small/medium rooms with moderate to high damping, going beyond the validity of the PWRC. SWRCs, either the Chien/Soroka approximation or an exact solution, keep the results quite close to a full wave model. Therefore, models 2, 3, or 4 are quite relevant in the design of classrooms and offices, which require relatively short reverberation times.

For rooms with longer reverberation times, the calculations can become tedious when using an SWRC, because more beams as well as more reflections will be required. The solution to this problem is to treat the image sources with large propagation distance in a statistical manner. This is acceptable, because larger propagation distances correspond to delay times that fall in the reverberant tail of an impulse response, which can be modeled with statistical approaches. ${ }^{29,30}$ Therefore, it may be more practical to use an exact half-space solution for early reflections only, especially since their perceptual effect is more important relative to late reverberation. ${ }^{30}$

\section{CONCLUDING REMARKS}

Two exact, half-space solutions are incorporated into a phased, approximate beam tracing model in order to predict the SPL in rectangular rooms up to three times the Schroeder frequency. Using an exact half-space solution reduces the error of the PWRC by 1 or $2 \mathrm{~dB}$ over a large frequency 
range. Although some of the remaining error may be attributed to the scanning error, this work shows that much of the error in phased GA can be eliminated by use of an SWRC.

Geometrical acoustics methods give higher errors for longer source-to-receiver distances, because lower direct-toreverberant energy ratios are coupled with less accurate calculation of the reflected pressures. This effect is greatly reduced by use of an SWRC, because small $\psi$ and large $\phi$ or $\alpha$ are properly taken into account.

SWRCs require more reflections, in general, and involve numerical integration or the calculation of an infinite series. This computational burden is eased by implementing frequency-dependent stopping criteria so that higher order reflections are not calculated for frequencies that have already converged.

Errors at off-resonances using the PWRC can be large enough that band-averaged errors can reach $1 \mathrm{~dB}$ or more, even above the Schroeder frequency. In this context, a crossover frequency for interference effects can be defined as the band-averaged difference in SPL when using the PWRC or an SWRC. A simple analysis shows that the crossover frequency varies greatly among different rooms and can be as high as 5 or $6 f_{S}$.

\section{ACKNOWLEDGMENTS}

The Research Fund KU Leuven, the Fund for Scientific Research - Flanders (F.W.O.) - and Flanders Make, the strategic research centre for the manufacturing industry, are gratefully acknowledged for support of this research.

${ }^{1}$ H. Kuttruff, Room Acoustics, 5th ed. (Spon Press, Abingdon, England, 2009), $101 \mathrm{pp}$.

${ }^{2} \mathrm{~T}$. Lewers, "A combined beam tracing and radiant exchange computer model of room acoustics," Appl. Acoust. 38, 161-178 (1993).

${ }^{3}$ C.-H. Jeong, "Absorption and impedance boundary conditions for phased geometrical-acoustics methods," J. Acoust. Soc. Am. 132(4), 2347-2358 (2012).

${ }^{4}$ Y. W. Lam, "Issues for computer modelling of room acoustics in nonconcert hall settings," Acoust. Sci. Tech. 26(2), 145-155 (2005).

${ }^{5}$ J. S. Suh and P. A. Nelson, "Measurement of transient response of rooms and comparison with geometrical acoustic models," J. Acoust. Soc. Am. 105(4), 2304-2317 (1999).

${ }^{6} \mathrm{M}$. Ochmann, "The complex equivalent source method for sound propagation over an impedance plane," J. Acoust. Soc. Am. 116(6), 3304-3311 (2004).

${ }^{7}$ F. P. Mechel, Formulas of Acoustics, 2nd ed. (Springer-Verlag, Berlin, 2008), $176 \mathrm{pp}$.

${ }^{8}$ A. Sommerfeld, "Über die ausbreitung der wellen in der drahtlosen telegraphie" ("On the propagation of waves in wireless telegraphy"), Ann. Phys. 333, 665-737 (1909).
${ }^{9}$ J. E. Piercy and T. F. W. Embleton, "Review of noise propagation in the atmosphere," J. Acoust. Soc. Am. 61(6), 1403-1418 (1977).

${ }^{10}$ J. A. Fawcett, "Complex-image approximations to the half-space acoustoelastic Green's function,” J. Acoust. Soc. Am. 108(6), 2791-2795 (2000).

${ }^{11}$ G. Taraldsen, "The complex image method," Wave Motion 43, 91-97 (2005).

${ }^{12} \mathrm{G}$. Taraldsen, "A note on reflection of spherical waves," J. Acoust. Soc. Am. 117(6), 3389-3392 (2005).

${ }^{13} \mathrm{M}$. Gensane and F. Santon, "Prediction of sound fields in rooms of arbitrary shape: Validity of the image sources method," J. Sound Vib. 63(1), 97-108 (1979).

${ }^{14}$ S. M. Dance, J. P. Roberts, and B. M. Shield, "Computer prediction of sound distribution in enclosed spaces using an interference pressure model," Appl. Acoust. 44, 53-65 (1995).

${ }^{15}$ E. De Geest and R. Garcea, "Simulation of room transmission functions using a triangular beam tracing computer model," in IEEE Workshop on Applications of Signal Processing to Audio and Acoustics (WASPAA, 1995), New Paltz, NY (October 15-18, 1995).

${ }^{16}$ E. De Geest and H. Patzold, "Comparison between room transmission functions calculated with a boundary element method and a ray tracing method including phase," in Inter-noise 96, Liverpool, UK (July 30 to August 2, 1996), pp. 3177-3180.

${ }^{17}$ M. Aretz, P. Dietrich, and M. Vorländer, "Application of the mirror source method for low frequency sound prediction in rectangular rooms," Acta Acust. Acust. 100(2), 306-319 (2014).

${ }^{18}$ A. Wareing and M. Hodgson, "Beam-tracing model for predicting sound fields in rooms with multilayer bounding surfaces," J. Acoust. Soc. Am. 118(4), 2321-2331 (2005).

${ }^{19} \mathrm{~K}$. M. Li and P. M. Lam, "Prediction of reverberation time and speech transmission index in long enclosures," J. Acoust. Soc. Am. 117(6), 3716-3726 (2005).

${ }^{20}$ X. Di and K. E. Gilbert, "An exact Laplace transform formulation for a point source above a ground surface," J. Acoust. Soc. Am. 93(2), 714-720 (1993).

${ }^{21}$ The MathWorks Inc., MATLAB, version R2015a (Natick, MA, 2015).

${ }^{22}$ S. Marburg and B. Nolte, Computational Acoustics of Noise Propagation in Fluids - Finite and Boundary Element Methods (Springer-Verlag, Berlin, Germany, 2008), 579 pp.

${ }^{23} \mathrm{H}$. Bériot, A. Prinn, and G. Gabard, "Efficient implementation of highorder finite elements for Helmholtz problems," Int. J. Numer. Meth. Engng. 106, 213-240 (2016).

${ }^{24}$ C.-H. Jeong, D. Lee, S. Santurette, and J.-G. Ih, "Influence of impedance phase angle on sound pressures and reverberation times in a rectangular room," J. Acoust. Soc. Am. 135(2), 712-723 (2014).

${ }^{25} \mathrm{G}$. A. Miller, "Sensitivity to changes in the intensity of white noise and its relation to masking and loudness," J. Acoust. Soc. Am. 19, 609-619 (1947).

${ }^{26} \mathrm{C}$.-H. Jeong and J.-G. Ih, "Effects of source and receiver locations in predicting room transfer functions by a phased beam tracing method," J. Acoust. Soc. Am. 131(5), 3864-3875 (2012).

${ }^{27} \mathrm{M}$. Vorländer, "Computer simulations in room acoustics: Concepts and uncertainties," J. Acoust. Soc. Am. 133(3), 1203-1213 (2013).

${ }^{28}$ C.-H. Jeong, "Improved low frequency room responses by considering finiteness of room boundary surfaces," Acta Acust. Acust. 99, 545-556 (2013).

${ }^{29}$ D. van Maercke and J. Martin, "The prediction of echograms and impulse responses within the Epidaure Software," Appl. Acoust. 38, 93-114 (1993).

${ }^{30}$ G. M. Naylor, "ODEON-another hybrid room acoustical model," Appl. Acoust. 38, 131-143 (1993). 